\title{
Low Momentum Direct Photon Measurement
}

\author{
Wenqing Fan for PHENIX collaboration ${ }^{1, a}$ \\ ${ }^{1}$ Stony Brook University
}

\begin{abstract}
Direct photons have long been considered as golden probes to study the properties of the Quark Gluon Plasma (QGP). They do not interact strongly with the medium and are produced at all stages of the collision, hence carrying information of the entire evolution of the system to the detectors. The PHENIX experiment discovered a large excess of low $p_{T}$ photons in $\mathrm{Au}+\mathrm{Au}$ collisions at $\sqrt{s_{N N}}=200 \mathrm{GeV}$ compared to reference $\mathrm{p}+\mathrm{p}$ collisions, which has been interpreted as thermal radiation from the QGP and hadron gas medium. At the same time, the excess photons show a large elliptic and triangular flow. These results are challenging for the current theoretical models to describe simultaneously, because on one hand the large yield suggests early stage emissions when the temperature is high, on the other hand the large anisotropy is expected to be formed only at later stages of the collision when the system has cooled off and the thermal photon production rate is expected to be smaller. Using a variety of high statistics datasets across different collision systems and energies in PHENIX, simultaneous analyses of yields and azimuthal asymmetries of direct photons with higher precision are performed to provide more constraints to the theoretical calculations. In this talk, we will present recent results on low $p_{T}$ direct photons measured via their external conversions to electron-positron pairs, including new results from $\mathrm{Au}+\mathrm{Au}$ at lower beam energies of 39 and $62.4 \mathrm{GeV}$, as well as $\mathrm{Cu}+\mathrm{Cu}$ at $200 \mathrm{GeV}$.
\end{abstract}

\section{Introduction}

Photons are one of the most important probes to study the extremely dense and hot medium, Quark Gluon Plasma (QGP), created in the relativistic heavy ion collisions. Unlike hadronic probes, they do not strongly interact with the medium, hence leaving the medium unscathed and carrying the information of its space-time creation point. Also, photons are produced throughout every stage of the collision, which then enable us to access the whole dynamic evolution of the medium. Since our main interest is the property of QGP, the photons from hadronic decays are subtracted, and that is called "direct photon".

With direct photons, we could measure the thermal radiation to probe the temperature of the medium. In analogy to black body radiation, the spectrum of the thermal radiation, real photons or low mass lepton pairs, will reflect the temperature of the system. In addition, taking into account that it is a "moving source", the spectrum will also be related to how the system expands. Another important observable we could measure to study QGP is the collective behavior. In the hydrodynamics model,

a e-mail: wenqing.fan@stonybrook.edu 
QGP is a strongly coupled medium acting like a "perfect fluid". The droplet of QGP will expand differently under the different pressure gradient along different directions, and in the end evolve into the final state anisotropy, which is quantified by the Fourier decomposition of the azimuthal distribution of particles [1].

PHENIX has measured the direct photon yield and anisotropy in Au+Au collisions at RHIC energies. On one hand, a large excess of direct photons is observed at low $p_{T}$, and the yield exhibits strong centrality dependence [2]. On the other hand, a large anisotropy is also seen for those direct photons [3]. These two observations are very challenging to reconcile in the existing theoretical pictures, because a large yield indicates early stage emissions when the temperature is still high while a large anisotropy indicates late stage emissions when the collective flow is sufficiently built up. The failure to simultaneously explain the large yield and anisotropy is usually referred as "direct photon puzzle".

To provide more constraints to the theoretical models and more insights into the "direct photon puzzle", PHENIX has performed a systematic direct photon measurements across different collision systems, $\mathrm{Au}+\mathrm{Au}$ and $\mathrm{Cu}+\mathrm{Cu}$, and also different energies, from $39 \mathrm{GeV}$ to $200 \mathrm{GeV}$. We have also developed a new photon reconstruction and recognition technique to analyze the conversion photons at the layers of Silicon Vertex Tracker (VTX), which will enable us to reach a better precision given the high statistics. And with a variety of datasets available in PHENIX, we will explore more collision systems for the future measurements.

\section{Experiment Setup and Photon Measurement Methods}

The detector involved in the results presented in this paper is central arm of PHENIX detector, which has a $\pi / 2$ azimuthal coverage in the east and west arm separately, and a -0.35 to 0.35 pseudo-rapidity coverage, as depicted in figure 1. The central arm is mainly consist of three parts, tracking system to measure the trajectories and momenta of charged particles, the electromagnetic calorimeter (EMCal) to determine the energy photons and electrons, and the Ring Imaging Cerenkov detector (RICH) for the electron identification.

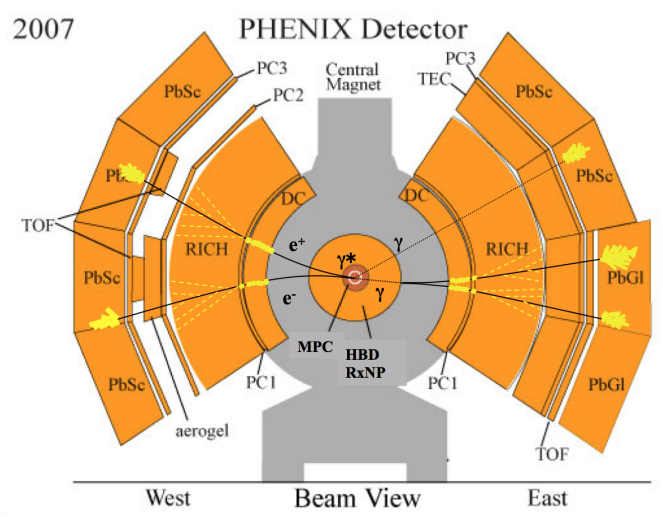

Figure 1. Schematic view of the PHENIX detector in PHENIX (2007). Dashed line corresponds to photon trajectory, solid curve corresponds to $e^{ \pm}$trajectory.

There are three independent methods used in PHENIX to measure photons. Historically we start with the calorimeter method where photons are reconstructed from their energy deposit in EMCal 
[4]. The calorimeter method gives a good resolution at high $p_{T}$ but struggles with the hadron contamination in the low $p_{T}$ region. To increase the photon purity at low $p_{T}$ side, conversion methods are developed where we reconstruct photons which convert to electron positron pairs. There are two kinds of conversion methods investigated here, one is the internal conversion which focuses on the virtual photons [5] and the other is the external conversion method which focuses on the real photons. convert at the detector materials [6].

$\mathrm{The} \mathrm{Cu}+\mathrm{Cu}$ results shown in the next section uses the internal conversion method [5]. In general, any source of high energy real photons can also emit virtual photons that materialize into $e^{+} e^{-}$pairs. And in the limit of $m_{e e} \ll p_{T}$, the $e^{+} e^{-}$pair production is related to the real photon production [7] as follows:

$$
\frac{d N_{e e}}{d m_{e e} d p_{T}} \simeq \frac{2 \alpha}{3 \pi} \frac{1}{m_{e e}} \frac{d N_{\gamma}}{d p_{T}}
$$

where $\alpha$ is the fine structure constant, $m_{e e}$ is the mass of $e^{+} e^{-}$pair. To extract the direct photon yield, all $e^{+} e^{-}$pairs between $0.12<m_{e e}<0.3 \mathrm{GeV} / c^{2}$ are fitted to a two-component fitting function defined below

$$
f_{\text {incl }}\left(m_{e e}\right)=\left(1-r_{\gamma}\right) f_{c}\left(m_{e e}\right)+r_{\gamma} f_{d i r}\left(m_{e e}\right)
$$

where $f_{c}\left(m_{e e}\right)$ is the cocktail mass distribution, $f_{d i r}\left(m_{e e}\right)$ is the expected shape of the virtual direct photon internal conversion mass distribution. $r_{\gamma}$ is the only fit parameter, which corresponds to the the direct photon fraction in the inclusive photon spectrum. Together with the inclusive photon yield, one could obtain the direct photon yield using

$$
d N^{d i r}\left(p_{T}\right)=r_{\gamma} d N^{i n c l}\left(p_{T}\right)
$$

The advantage of internal conversion method is that it gives a small background, however the signal is also small due to the small production rate.

The external conversion method [6] has more statistics as compared to the internal conversion method, and that is used in the new $\mathrm{Au}+\mathrm{Au}$ results shown here. We selected the real photons that are converted at the Hadron Blind Detector (HBD) [8] backplane, which located at a radius of $\sim 60$ $\mathrm{cm}$ and with $\mathrm{a} \sim 2.0 \%$ radiation length. The conversions are identified by the invariant mass of $e^{+} e^{-}$pairs. The standard track reconstruction algorithm assumes all tracks originating from the event vertex, which will result in an artificial $m_{e e} \sim 12 \mathrm{MeV}$ for these off-vertex conversions. An alternate tracking algorithm was then applied, which assumes that all tracks originating from HBD backplane and therefore the conversions will have an invariant mass $m_{e e}$ close to zero. By applying cuts on the two $m_{e e}$ from different algorithms, a conversion photon sample with purity $\sim 99 \%$ is reached. From that photon sample, we could obtain the direct photon yield using the following formula:

$$
d N^{d i r}\left(p_{T}\right)=\left(R_{\gamma}-1\right) d N^{\text {hadron }}\left(p_{T}\right)
$$

where $R_{\gamma}$ is defined as the ratio of inclusive photons and hadronic decay photons, and it is measured via the double ratio tagging method [6].

$$
R_{\gamma}=\frac{\gamma^{\text {incl }}}{\gamma^{\text {hadron }}}=\frac{\left\langle\epsilon_{\gamma} f\right\rangle\left(\frac{N^{\text {incl }}}{N^{\pi^{0}} \text { tag }}\right)_{\text {Data }}}{\left(\frac{\gamma^{\text {hadron }}}{\gamma^{\pi^{0}}}\right)_{\text {Sim }}}
$$

Here $\left\langle\epsilon_{\gamma} f\right\rangle$ is the conditional acceptance for pion tagging, $\left(\frac{N^{\text {incl }}}{N^{\pi^{0}} \text { tag }}\right)_{\text {Data }}$ is measured from data, and the denominator is obtained from simulation. 


\section{Results}

\subsection{Direct Photon Yield in Different Systems and Beam Energies}

The invariant yield of direct photons is measured in $\mathrm{Au}+\mathrm{Au}$ and $\mathrm{Cu}+\mathrm{Cu}$ collisions at $\sqrt{s_{N N}}=200$ $\mathrm{GeV}$. And the results are compared with our published results in $\mathrm{Au}+\mathrm{Au}$ collisions at the same collision energy. Figure 2 shows the direct photon $p_{T}$ spectra for minimum bias and our previous published $\mathrm{Au}+\mathrm{Au}$ data from [2] and [4]. Good consistency is observed for the three independent Au+Au measurements. Also shown in figure 2 are our $\mathrm{p}+\mathrm{p}$ data at $\sqrt{s_{N N}}=200 \mathrm{GeV}$ together with a empirical fit function in black dashed curve. The direct photon contribution from prompt processes could be estimated by scaling the $\mathrm{p}+\mathrm{p}$ fit with corresponding average number of binary collisions $N_{\text {coll }}$ in $\mathrm{Au}+\mathrm{Au}$ collisions, which is represented by the solid curve. While the direct photon production follows the $N_{\text {coll }}$ scaled $\mathrm{p}+\mathrm{p}$ curve at high $p_{T}$, a significant excess above the expected prompt production is observed below $3 \mathrm{GeV} / c$. The excess is attributed to the thermal radiation from the medium, and could be described by an exponential function with the inverse slope $T_{\mathrm{eff}} \approx 240 \mathrm{MeV} / c$.

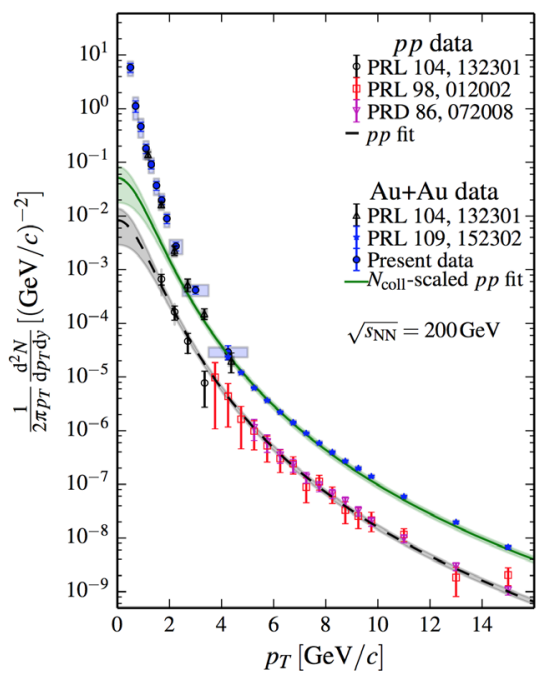

Figure 2. Direct photon $p_{T}$ spectra for minimum bias $\mathrm{Au}+\mathrm{Au}$ and $\mathrm{p}+\mathrm{p}$ collisions at $\sqrt{s_{N N}}=200 \mathrm{GeV}$.

Similar excess is also observed in $\mathrm{Cu}+\mathrm{Cu}$ system at $\sqrt{s_{N N}}=200 \mathrm{GeV}$, the results for minimum bias and central collisions are shown in figure 3. The black points represent the $\mathrm{Cu}+\mathrm{Cu}$ data and the blue curve is the $N_{\text {coll }}$ scale $\mathrm{p}+\mathrm{p}$ fit. The inverse slope is $T_{\text {eff }}=288 \pm 49($ stat $) \pm 50($ sys $)$ for minimum bias, which is consistent with the $\mathrm{Au}+\mathrm{Au}$ result within large uncertainty.

In addition of different collision species, we also obtained new results for different beam energies. Figure 4 shows the direct photon $p_{T}$ spectra for minimum bias $\mathrm{Au}+\mathrm{Au}$ collisions at 39 and $62.4 \mathrm{GeV}$. Clear direct photon signal is observed in these lower beam energies, and the inverse slopes are $T_{\text {eff }} \approx$ $211 \mathrm{MeV} / c$ for $62.4 \mathrm{GeV}$ and $T_{\text {eff }} \approx 177 \mathrm{MeV} / c$ for $39 \mathrm{GeV}$ respectively.

Figure 5 shows the dependence of inverse slope $T_{\text {eff }}$ on collision energy after combing our results together with an ALICE direct photon measurement of $\mathrm{Pb}+\mathrm{Pb}$ collisions at $\sqrt{s_{N N}}=2760 \mathrm{GeV}$ [9]. A possible increase of $T_{\text {eff }}$ with beam energy is seen, but it is not yet conclusive due to the large uncertainties in the plot. 

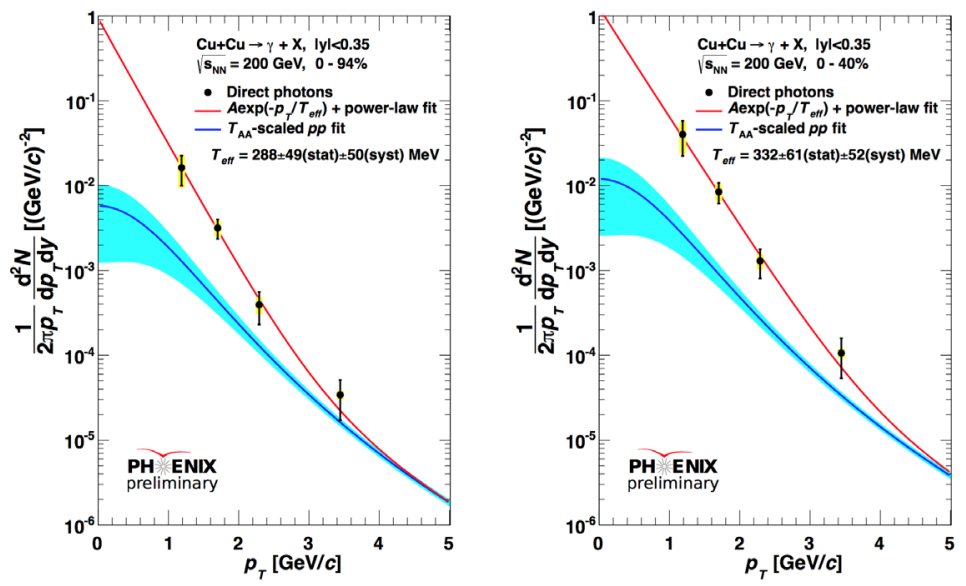

Figure 3. Direct photon $p_{T}$ spectra for minimum bias and central $\mathrm{Cu}+\mathrm{Cu}$ collisions at $\sqrt{s_{N N}}=200 \mathrm{GeV}$.
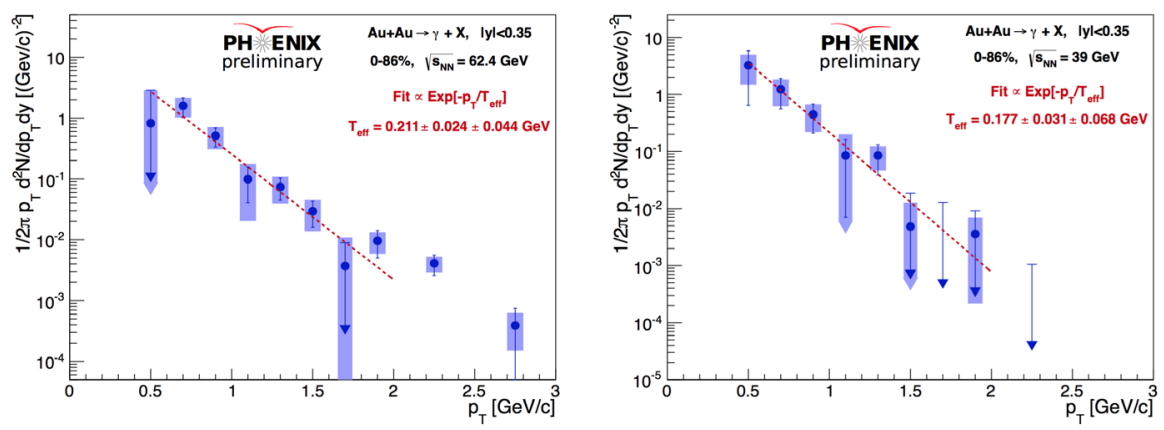

Figure 4. Direct photon $p_{T}$ spectra for minimum bias $\mathrm{Au}+\mathrm{Au}$ collisions at $\sqrt{s_{N N}}=39 \mathrm{GeV}$ and $62.4 \mathrm{GeV}$.

\subsection{Integrated Invariant Yield versus multiplicity}

To look further into the origin of the thermal radiation, the direct photon contribution from prompt processes is subtracted and the results are depicted in the left panel of figure 6 for $\mathrm{Au}+\mathrm{Au}$ collisions at $\sqrt{s_{N N}}=200 \mathrm{GeV}$ [6]. A clear increase of yield with centrality is observed, and the increase is quantified by the integrated yield above a threshold $p_{T}^{\min }$. As the centrality is directly related to $N_{\text {part }}$, the increase of the integrated yield is plotted as a function of $N_{\text {part }}$, shown in right panel of figure 6. The plot shows a linear rise of the yield with $N_{\text {part }}$ in the logarithmic representation and hence is fitted by power law function $A N_{\text {part }}^{\alpha}$ in dashed curve. From that we can see the yield scales with $\alpha=1.38 \pm 0.03$ (stat) \pm 0.07 (sys), which indicates that it increase faster than the reaction volume.

Another important observable is charged particle multiplicity $d N_{\mathrm{ch}} / d \eta$, because it relates to total energy of collision system. Similar interesting scaling behavior appears when we studied the $d N_{\mathrm{ch}} / d \eta$ dependence of the direct photon yield, and the results are illustrated in figure 7. Different data points correspond to different collision systems and energies. The green dashed curve is a power law function $A N_{\text {part }}^{\alpha}$ fitted to $\mathrm{Au}+\mathrm{Au}, \mathrm{Cu}+\mathrm{Cu}$ and $\mathrm{Pb}+\mathrm{Pb}$ data. We could see that the direct photon production in 


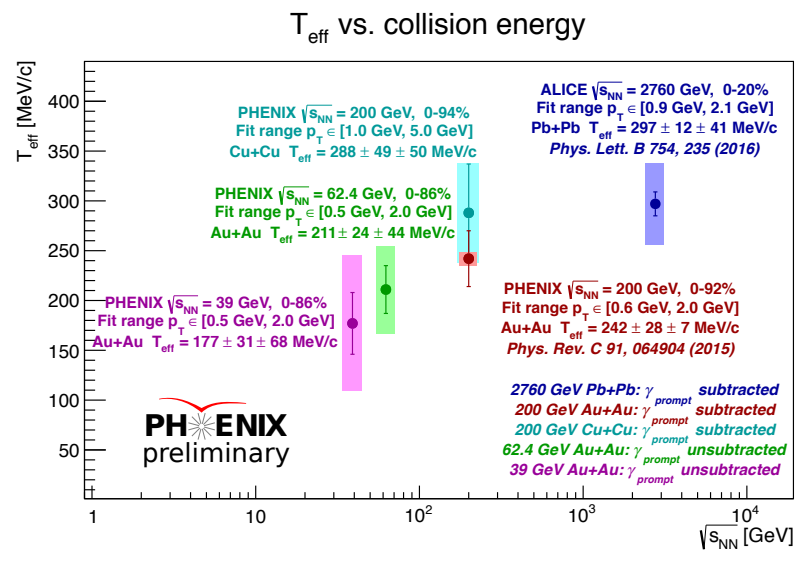

Figure 5. Inverse slope $T_{\text {eff }}$ versus collision energy $\sqrt{s_{N N}}$ for different systems, where $\gamma_{\text {prompt }}$ represents the prompt photon production estimated from reference $\mathrm{p}+\mathrm{p}$ measurement. $\gamma_{\text {prompt }}$ is subtracted for $\mathrm{Pb}+\mathrm{Pb}$ at 2760 $\mathrm{GeV}, \mathrm{Au}+\mathrm{Au}$ at $200 \mathrm{GeV}$ and $\mathrm{Cu}+\mathrm{Cu}$ at $200 \mathrm{GeV}$ data, but not subtracted for $\mathrm{Au}+\mathrm{Au}$ at $39 \mathrm{GeV}$ and $62.4 \mathrm{GeV}$ data because of the lack of the $\mathrm{p}+\mathrm{p}$ measurement at the corresponding beam energies at RHIC.

heavy ion collisions scales with $d N_{\mathrm{ch}} / d \eta$ from RHIC energy to LHC energy, and the scaling factor extracted from fitting is $\alpha=1.196 \pm 0.052$. Surprisingly, the prompt photons turn out to follow nearly the same scaling trend with $\alpha=1.217 \pm 0.092$, which is represented by the pink dashed fitting curve through $\mathrm{p}+\mathrm{p}$ data in figure 7 . Although the mechanism behind this scaling behavior is still under investigation, it could shed more light on the theoretical picture for the direct photon production.

\subsection{Azimuthal and Triangular Flow}

The event plane method is used for the flow measurement $[1,10]$. We measure the azimuthal distribution of photons with respect to the event plane $\Psi_{n}$, which is determined by the inner and outer Reaction Plane Detectors $(\mathrm{RxN})[11] . v_{n}$ are then obtained from the coefficients of Fourier decomposition

$$
\frac{d N}{d\left(\phi-\Psi_{n}\right)} \propto 1+\sum_{n} 2 v_{n} \cos \left(n\left(\phi-\Psi_{n}\right)\right)
$$

The direct photon $v_{2}^{d i r}$ and $v_{3}^{d i r}$ are calculated from the inclusive photon $v_{n}^{\text {inc }}$ and the (simulated) decay photon $v_{n}^{d e c}$, as well as the ratio $R_{\gamma}$ of the total inclusive and decay photon yields, using the formula of [4]:

$$
v_{n}^{d i r}=\frac{R_{\gamma} v_{n}^{i n c}-v_{n}^{d e c}}{R_{\gamma}-1}
$$

Figure 8 shows the results for direct photon $v_{2}^{d i r}$ and $v_{3}^{d i r}$ obtained for three centralities using two separate methods for photon sample selection. The two different methods cover slightly different $p_{T}$ ranges, and the results are consistent in the overlap region. From figure 8, a large $v_{2}^{\text {dir }}$ is observed with a strong centrality dependence. $v_{2}^{d i r}$ appears to converge toward zero at high $p_{T}$. A sizable $v_{3}^{d i r}$ is observed as well, but there is no clear centrality dependence in this case. 


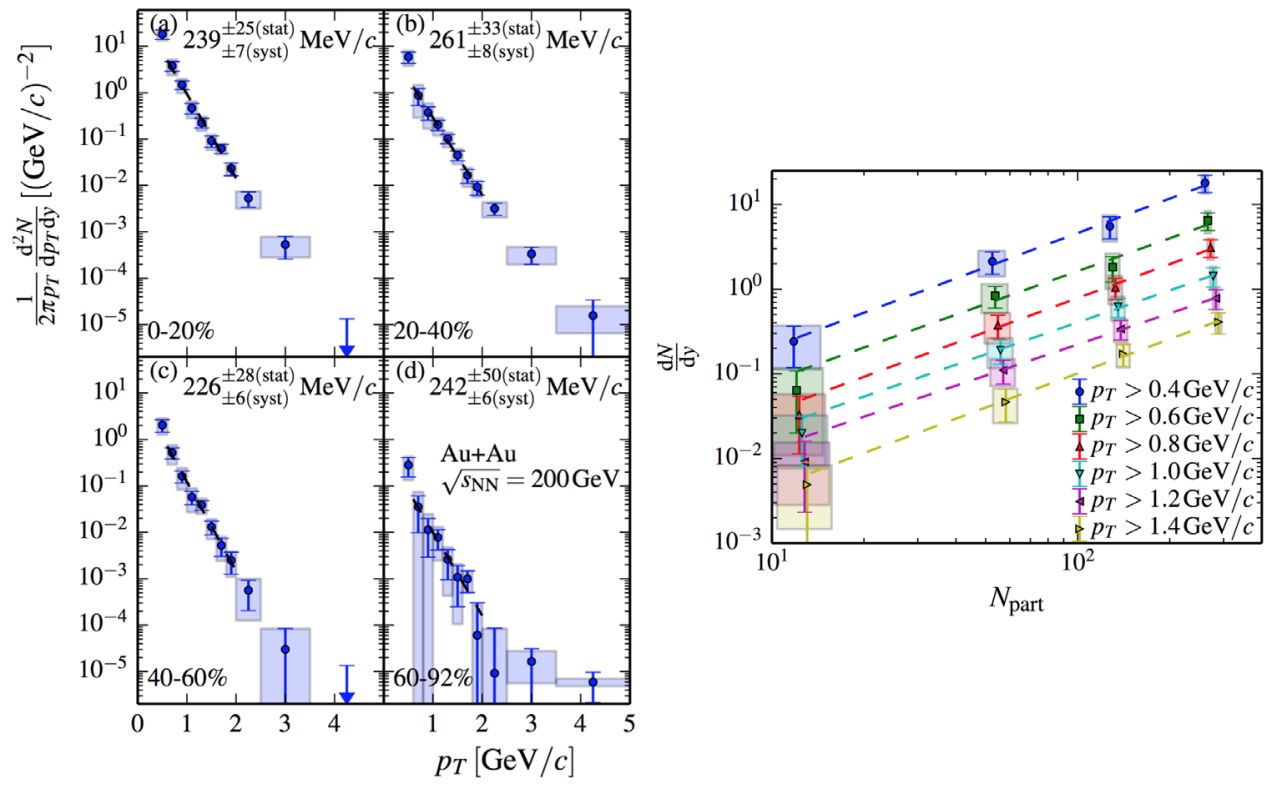

Figure 6. Left: Direct photon $p_{T}$ spectra after subtraction of the $N_{\text {coll }}$ scaled $\mathrm{p}+\mathrm{p}$ contribution in centrality bins 0 $20 \%, 20 \%-40 \%, 40 \%-60 \%, 60 \%-92 \%$. Right: Integrated thermal photon yields as a function of $N_{\text {part }}$ for different lower $p_{T}$ integration limits.

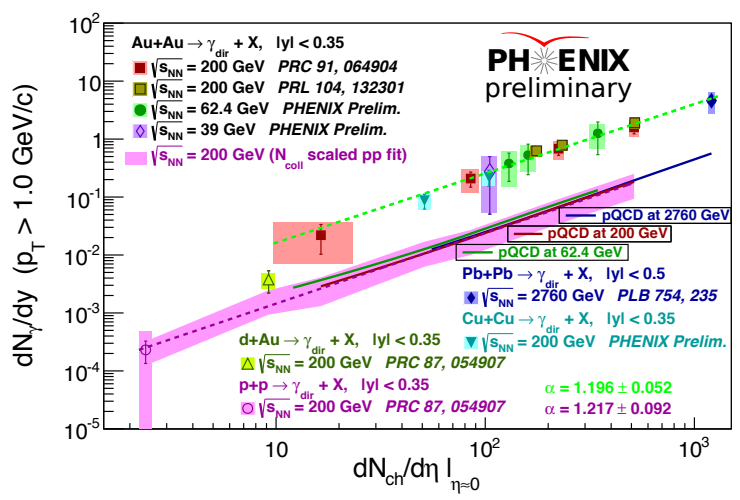

Figure 7. Integrated direct photon yield versus charged particle multiplicity $\left(p_{T}\right.$ integrated from $1 \mathrm{GeV} / c$ )

With the yield and flow we measured for $\mathrm{Au}+\mathrm{Au}$ collisions at $\sqrt{s_{N N}}=200 \mathrm{GeV}$, we compared our data with several theoretical models [12-15] in figure 9. What we found is that none of the models shown here could describe the large yield and large elliptic flow $v_{2}$ simultaneously to a satisfying level. 


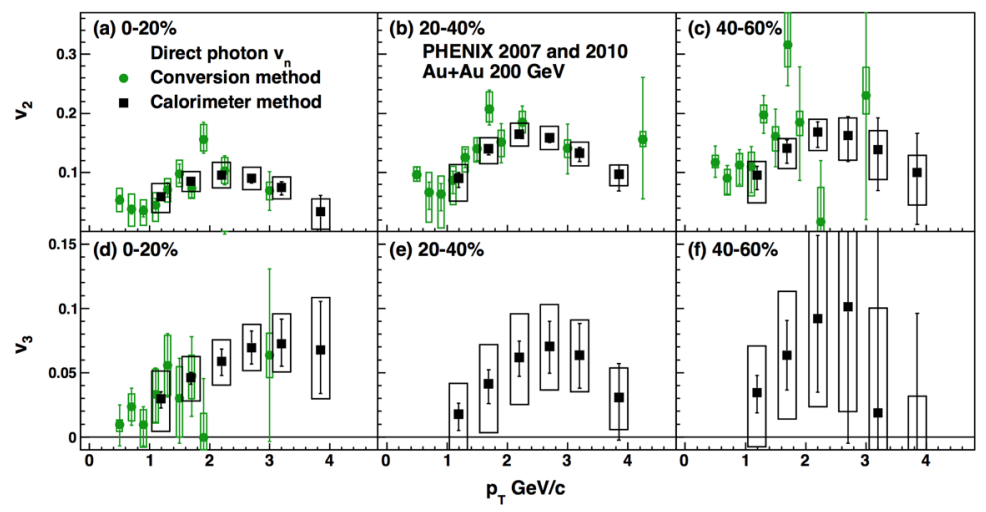

Figure 8. Direct photon $v_{2}$ and $v_{3}$ for $\mathrm{Au}+\mathrm{Au}$ collisions at $\sqrt{s_{N N}}=200 \mathrm{GeV}$ in $0-20 \%, 20 \%-40 \%, 40 \%-60 \%$ centrality bins.
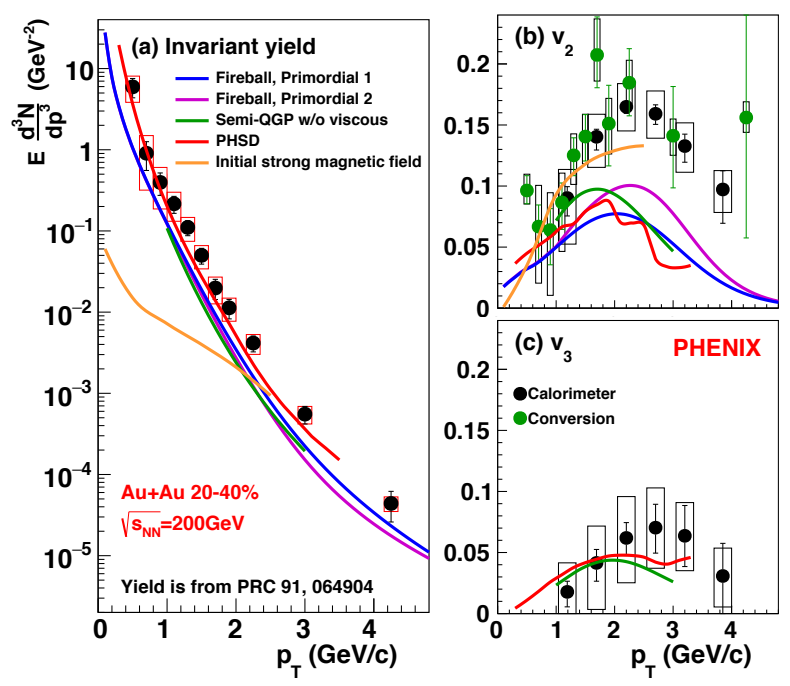

Figure 9. Comparison of the direct photon yield and $v_{2}$ to theoretical models.

\section{Summary}

To summarize, PHENIX has measured both yields and azimuthal anisotropy of low $p_{T}$ direct photons in $\mathrm{Au}+\mathrm{Au}$ collisions at $\sqrt{s_{N N}}=200 \mathrm{GeV}$. A large enhancement above the reference $\mathrm{p}+\mathrm{p}$ collisions is observed below $3 \mathrm{GeV} / c$. As for the flow measurement, large $v_{2}^{\text {dir }}$ and $v_{3}^{\text {dir }}$ are observed using two independent methods - external conversion method and calorimeter method. While $v_{2}^{\text {dir }}$ exhibits a clear dependence on centrality, such dependence is not seen in the $v_{3}^{d i r}$ result. Several theoretical model comparisons are made for both the yield and flow measurements, however the models fail to describe the large yield and large anisotropy simultaneously. PHENIX has also measured the low $p_{T}$ direct spectra in $\mathrm{Cu}+\mathrm{Cu}$ collisions at at $\sqrt{s_{N N}}=200 \mathrm{GeV}$, and $\mathrm{Au}+\mathrm{Au}$ collisions at $\sqrt{s_{N N}}=39$ and 
62.4 GeV. Clear direct photon signals are seen in every system. After combing different PHENIX data together with an additional ALICE data in $\mathrm{Pb}+\mathrm{Pb}$ collisions at $2760 \mathrm{GeV}$, the inverse slope $T_{\text {eff }}$ shows a hint of increase with collision energy. We also observed a scaling behavior of direct photon production with charge particle multiplicity $d N_{\mathrm{ch}} / d \eta$ in heavy ion collisions from RHIC to LHC energy. To further investigate the scaling behavior of direct photon production and provide more input to resolve "direct photon puzzle", systematic studies using a variety of high statistics datasets across different collision systems and energies are ongoing in PHENIX.

\section{References}

[1] A. M. Poskanzer and S. A. Voloshin, Phys. Rev. C 58, 1671 (1998).

[2] A. Adare et al. (PHENIX Collaboration), Phys. Rev. Lett. 104, 132301 (2010)

[3] A. Adare et al. (PHENIX Collaboration), Phys. Rev. C 94, 064901 (2016)

[4] A. Adare et al. (PHENIX Collaboration), Phys. Rev. Lett. 109, 152302 (2012)

[5] A. Adare et al. (PHENIX Collaboration), Phys. Rev. C 81, 034911 (2010)

[6] A. Adare et al. (PHENIX Collaboration), Phys. Rev. C 91, 064904 (2015).

[7] P. Lichard, Phys. Rev. D 51, 6017 (1995).

[8] W. Anderson et al., Nucl. Inst. Methods Phys. Res., Sect. A A646, 35 (2011).

[9] J. Adam et al., Phys. Lett. B 754, 235-248 (2016).

[10] S. Afanasiev et al. (PHENIX Collaboration), Phys. Rev. C 80, 024909 (2009).

[11] Stankus, Paul W., Milan Matos, and PHENIX Collaboration, Nuclear Instruments and Methods in Physics Research Section A: Accelerators, Spectrometers, Detectors and Associated Equipment 636.1 (2011).

[12] van Hees, Hendrik, Charles Gale, and Ralf Rapp, Phys. Rev. C 84, 054906 (2011).

[13] C. Gale, Y. Hidaka, S Jeon, S. Lin, J.-F. Paquet, R. D. Pisarski, D. Satow, V. V. Skokov, and V. Vujanovic, Phys. Rev. Lett. 114, 072301 (2015).

[14] O. Linnyk, W. Cassing, and E. L. Bratkovskaya, Phys. Rev. C 89, 034908 (2014).

[15] G. Basar, D. E. Kharzeev, V. Skokov, Phys. Rev. Lett. 109, 202303 (2012). 